\title{
Second cancers after radiotherapy: update and recommandations
}

\author{
J.-M. Cosset ${ }^{1, *}$, a, M. Hetnal ${ }^{2}$ and C. Chargari ${ }^{3,4}$ \\ ${ }^{1}$ Centre de cancérologie - GIE Charlebourg, Groupe Amethyst, 92250 La Garenne-Colombes, France. \\ 2 Amethyst Radiotherapy Centre, Rydygier Hospital, Krakow, Poland. \\ ${ }^{3}$ Institut Gustave-Roussy, 94600 Villejuif, France. \\ 4 École du Val-de-Grâce, French Military Health Academy, 75005 Paris, France.
}

Received: 15 February 2018 / Accepted: 11 April 2018

\begin{abstract}
While radio-induced cancers are well known since the first years of the xxth century, they did not represent a major concern for radiation oncologists for many decades. With better and better results of modern radiotherapy and prolonged follow-up of patients, secondary radio-induced cancers should now be systematically taken into account when irradiating patients. The analysis of the available literature allows to stress a few main points; (1) cancer patients are at a higher risk for developing secondary cancers than the general population, radiotherapy being only responsible for a (small) proportion of those second malignancies; (2) the clinical data emphasize the role of age, with children being much more susceptible to the carcinogenic effect of ionizing radiation than adults; (3) most radio-induced cancers occur in or close to the high-dose treatment volume; (4) the relative risk of radio-induced cancer appears to be different for different organs; (5) the relative risks of radio-induced cancers tend to be lower in the medical cohort studies than in the Japanese A-Bomb survivor studies; (6) several cofactors (genetic, lifestyle...) account for the risk of secondary and radiation-induced cancer. The exact shape of the dose/effect (carcinogenesis) curve is still debated, particularly for the high doses of radiotherapy, with a direct impact on risk calculations, which can be very different if using different radiobiological models. In spite of some uncertainties, a few main recommendations could be proposed to reduce as much as possible the risk of radio-induced cancer after radiotherapy: (1) adapting the irradiation technique; (2) reducing the target volumes; (3) adapting to patient's age; (4) adapting to specific organs; (5) and optimizing the imaging dose. In conclusion, even if radioinduced cancers are rare, they must be kept in mind each time a radiotherapy is proposed in 2018.
\end{abstract}

Keywords: radio-induced cancer / radiotherapy / carcinogenesis / radiosensivity

\section{Introduction}

Radio-induced cancers were detected very early in the history of Radiology and Radiotherapy: a first case was reported as soon as 1902 by Frieben, and subsequently, a number of pioneers paid a heavy tribute to their work with radiation: among them, Marie Curie and Irène Joliot-Curie, whose deaths (by myeloblastic aplasia and chronic myeloid leukemia, respectively) were clearly related to a whole life devoted to their study of radiations.

The "Monument to the X-ray and Radium Martyrs" unveiled in Hamburg, Germany, in 1936, commemorates those who died due to their work with the use of radiation. When unveiled, the memorial included 169 names; by 1959, there

\footnotetext{
*Corresponding author:

jean-marc. cosset@amethyst-radiotherapy.com

${ }^{\mathrm{a}}$ Former Vice-Chairman, ICRP Committee 3.
}

were 359 names, a number most probably largely underestimated.

The dramatic consequences of the Hiroshima and Nagasaki bombing, in August 1945, unfortunately achieved to prove, if necessary, the carcinogenic risks of high doses of ionizing radiation.

In spite of such an evidence, we have to recognize that, for decades, radiation oncologists did not consider the carcinogenic risk of their therapeutic irradiations as being a real topic of concern.

The relative lack of interest, for years, of radiation oncologists for the carcinogenic risk of the therapy they applied, is most probably due to the poor survival results of radiotherapy in the first half of the xxth century. Moreover, at that time, a patient surviving more than 5 years was usually considered as being cured, and his follow-up was stopped (although we now know that most radio-induced cancers are emerging more than 5, and even 10-15 years after irradiation). Even when the radiation oncologist was aware of the 
emergence of a second cancer, the current attitude was to consider it as linked to chance, or to genetic, or to the way of life (tobacco, alcohol...), and not related to the radiotherapy which was delivered to the patient.

It was only in the $1960 \mathrm{~s}-1970$ s that some pioneers clinically detected an excess of second cancers after radiotherapy. The first observations were coming in particular from cohorts of Hodgkin's disease patients, simply because radiation oncologists had available large series of patients - frequently young - cured for more than 5 years, and after the irradiation of large volumes (mantle fields, inverted Y) (Somers et al., 1990; Cosset et al., 1992, 2016). In the $1980 \mathrm{~s}$, the prominent role of the irradiated volume was demonstrated, as well as the higher risk of radio-induced cancers in young adults, and even more in children (Hall, 2006).

Today, in 2018, the carcinogenic risk of any radiotherapy has been extensively studied, and this risk must be kept in mind when deciding the therapy and when treating the patients. This risk must be taken into account even if, fortunately, it is usually very small (and even negligible in some cases).

Thus, the goal of this paper is to make the point on radioinduced cancers, to update our knowledge on second cancers after radiotherapy and to make recommandations to the radiation therapists.

\section{Second cancers; radio-induced or not?}

The wording "second cancer" needs to be clarified.

Cancer unfortunately remains a frequent pathology, and it is clear that to have been the victim of a cancer does not "protect" against a second one (Langballe et al., 2011). Moreover, the cause of the first cancer (genetic predisposition, or way of life - see above-) may remain, and may favor the emergence of a second one.

It is therefore difficult to identify the "second cancer" cases which can be considered as radio-induced, and those which are clearly not related to irradiation. Up to now, we do not have available, except in rare specific cases, any specific genetic mutations which would prove the radiogenic cause of a "second cancer".

The study of large cohorts shed some light on this problem: in the extensive 2007 review by Suit et al. (2007), the relative risk (RR) for a second primary cancer in 11 cohorts of cancer patients was as high as 1.31, when comparing the radiotherapy patients (RT) and the general population (GP): RR RT/ $\mathrm{GP}=1.31(95 \% \mathrm{CI} ; 1.15-1.49)$. It therefore appears that cancer survivors do have a higher risk of developing a "second cancer".

However, this relative risk of 1.31 does not appear to be only due to irradiation, since the RR for the non-irradiated cancer patients is found to be 1.12 (RR non-RT/GP). The real risk of radio-induced second cancers is therefore better evaluated by the RR: "RT/non-RT", which compares the cancer patients who received an irradiation to those that did not receive any irradiation. This relative risk RT/non-RT in the Suit's study is 1.08 (95\% CI: $1.00-1.17)$, with therefore a confidence interval overlapping the value 1 . This last RR "RT/non-RT" gives a better indication of the carcinogenic role (borderline significant) of radiotherapy (Suit et al., 2007).
Berrington de Gonzalez studied the proportion of second cancers attributable to radiotherapy treatment in adults, in a large cohort study of 647,672 cancer patients from the US Surveillance, epidemiology and end results (SEER) cancer registries (Berrington de Gonzalez et al., 2011). She concluded that a relatively small proportion of second cancers (about $8 \%$ of all "second cancers") are related to radiotherapy in adults, suggesting that most are due to other factors, such as lifestyle or genetics.

\section{Radio-induced cancers: lessons from the literature}

A huge number of reviews are now available in the literature, e.g., the recent document of the American association of Physicists in medicine (AAPM) (Kry et al., 2017). From this large body of literature and from our own work with an ICRP/ICRU task group (report unfortunately unpublished to date), a few main points can be highlighted:

- cancer patients are at a higher risk for developing secondary cancers than the general population, but - see above - radiotherapy is only responsible for a (small) proportion of the second malignancies (Suit et al., 2007; Berrington de Gonzalez et al., 2011);

- the clinical data emphasize the role of age, with children being much more susceptible to the carcinogenic effect of ionizing radiation than adults (a 3-6-fold increase) (Hall, 2006);

- the reviews of available data confirms the clinical experience, according to which "the majority of secondinduced cancers occur in or close to the high-dose treatment volume" (Hall, 2006; Cosset et al., 2016);

- the relative risk of cancer appears to be different for different organs, with the thyroid probably being the best example of an organ particularly sensitive to the carcinogenic effect of radiation, especially in children (Cosset et al., 2016);

- the relative risks of radio-induced cancers tend to be lower in the medical cohort studies than in the Japanese A-Bomb survivor studies (Little, 2001). The fractionation/protraction of most of the therapeutic irradiations, as well as the neutron component in the A-bomb data may account for this difference (Schneider and Walsh, 2008);

- several cofactors account for the risk of radiation-induced cancer. Those include lifestyle (e.g.: smoking habit), individual susceptibility (Foray et al., 2016; Colin et al., 2017), as well as chemotherapeutic agents (in particular alkylants agents) (Travis et al., 2002). Moreover, familial cancer syndromes should probably be considered as an additional risk for second radio-induced cancers (Schneider, 2011).

\section{Radiobiological models}

The dose/effect model (or risk model) to be used for radioinduction of cancers remains, in 2018, a burning and endless topic (Chargari et al., 2016; Cosset et al., 2016; Kry et al., 2017). 
A number of authors sticks to the Linear-No-Threshold (LNT) model, whatever the dose, thus from 0 Gy until the large doses of 70-80 Gy of radiotherapy.

The LNT model was mainly derived from the data observed after Hiroshima and Nagasaki, data which showed a clear linear relationship between 0.5 and a few grays. This is often considered as the "Gold standard", but:

- even in the dose range mentioned above, we previously saw that this model, based on atomic bomb survivors, could overestimate the risk in the medical radiotherapy series (Little, 2001);

- for low doses (below 0.5 Gy), radiobiologists are still fighting, some of them sticking to the LNT model, some others proposing a threshold (or at least a "practical" threshold, generally about $100 \mathrm{mSv}$ ), and finally, others describing an underestimation of the risk by the LNT model (particularly due to some "bystander effect")...

- for "high doses" (above a few grays, but with large variations from a study to another), three models are in competition; the LNT one (which would suggest a very high - unrealistic? - risk at high radiotherapy doses), the "bell-shaped" model, following data, some as old as 1957 by Gray himself (Gray, 1957), showing a decrease of the risk at high doses, and several "plateau" models, where the risk is "plateauing" after a certain dose. However, the exact dose level of the inflexion point (a few grays? $40 \mathrm{~Gy}$ ?) remains debated in 2018 and could depend on the organ at risk which is considered; for example, some data indicate a dramatic decrease of the risk at high doses for thyroid (Kry et al., 2017).

While the battle is not finished, one can only very prudently suggest that the presently available clinical data seem to support some "plateauing" at very high doses.

\section{Which recommandations in 2018 ?}

The ICRP/ICRU task group and the AAPM TG 158 document (Kry et al., 2017) proposed recommendations (actually almost identical) to try and reduce the risk of secondary radio-induced cancers after radiotherapy.

Today, radiation oncologists and physicists are well aware of the advantages of reducing the volumes irradiated at high doses, with more and more sophisticated techniques (IMRT, VMAT, Gating and tracking, IGRT,...). This had allowed a significant decrease of the deterministic effects and should also decrease the risk of second radio-induced cancers in the areas receiving high doses of radiation.

However, in parallel, it is imperative that those same professionals understand the magnitude of the dose levels outside of the treated volume, and are aware of methods to manage them.

The low doses outside the target volumes have been neglected for too long, and the profession is facing a new challenge: to reduce as much as possible the deterministic effects, and the carcinogenic risk close to the target volumes, while reducing at the same time the "low doses" far away from the treated volume, in order to avoid radio-induced cancers in those areas.

Actually, such a caveat had been well emphasized in ICRP publication 73, in the European Directive 97/43, and subsequently in the European Directive 2013/59: "For radiotherapeutic purposes, exposures of target volumes shall be individually planned; taking into account that doses of nontarget volumes and tissues shall be as low as reasonably achievable and consistent with the intended radiotherapeutic purpose of the exposure".

We are summarizing here the main recommandations for reducing the risk of radio-induced cancer after radiotherapy.

\subsection{Adapting the irradiation technique}

- AAPM TG 158 (Kry et al., 2017) well emphasized the trade-off of modern IMRT (intensity modulated radiotherapy) treatments, relative to $3 \mathrm{D}$ conformal radiotherapy (CRT). IMRT allows decreased treated volume through increased conformality, which is reducing the volume of tissue receiving a high dose. However, this is done at the cost of increased head leakage from increased number of Monitor units (MUs), which increases doses farther from the target, and thus the "integral dose" (the total energy absorbed by the body);

- the more recent VMAT (volumetric modulated arc therapy) technique is using less MUs, and therefore allows to reduce this integral dose;

- flattening filter: for both IMRT and stereotactic procedures, the out-of-field dose is reduced when the flattening filter is removed from the beam line, so FFF (flattening filter free) delivery is an improvement in terms of reduction of the integral dose;

- photon energy: here again, the radiation oncologist has to face a trade-off between high- and low-energy treatments. High-energy therapy is associated with some (fortunately low) neutron production. Low-energy photon therapy results in higher stray photon dose because of the greater number of MUs required. Although difficult to precisely quantify, the added neutron contamination at high energy seem to be offset by the added stray photon dose at low energy. For the AAPM TG 158, the optimal energy could be intermediate such as 10 MV (Kry et al., 2017);

- proton therapy allows a substantial reduction in dose distal to the target, resulting in reduced integral dose (typically by a factor of 2-3 compared with IMRT). The dosimetric advantage to the patient is even more pronounced when scanning proton therapy is employed. This technique improves the dose conformality relative to scattered proton therapy and thereby reduces the integral dose to the patient. It also reduces the dose associated with neutrons produced in the treatment head (AAPM TG 158 [Kry et al., 2017]);

- brachytherapy is probably the best irradiation modality for minimizing the risk of second cancer. Volumes receiving low to intermediate doses are decreased, and the theoretical risk of second cancer outside the radiation fields is lowered, as compared with IMRT techniques (Zelefsky et al., 2012).

\subsection{Reducing the target volumes}

- The larger the irradiated volume, the higher the risk of a secondary radio-induced cancer. This has been shown as early as 1990 by analyzing the data of the International 
database for Hodgkin's disease (HD) (Somers et al., 1990). Reducing the size of the clinical target voulme (CTV) or planning target volume (PTV) can be one of the most potent options for reducing the dose to non-target structures (Kry et al., 2017);

- in clinical practice, the CTV has already been reduced in many clinical situations. For Hodgkin's lymphoma, for example, the former large field irradiation has been replaced in most cases by the treatment of the involved regions only, with already a positive impact on the risk of radio-induced cancers (Tubiana, 2009; Koshy et al., 2012). In some other cases, the irradiation of some "prophylactic" lymph node areas could be omitted (for testicular, breast and prostate cancers, for example);

- reducing the PTV margin can be a simple way to decrease the irradiated volume, but one should keep in mind that such a margin reduction is typically associated with increased imaging (see below).

\subsection{Adapting to patient's age}

- As previously shown, children are much more prone than adults to develop a radio-induced second cancer after a given dose of irradiation. There is no "cut-off" for risk depending on age; the risk, being very high for the newborn, decreases progressively with age (Hall, 2006);

- in children, when irradiation cannot be omitted, everything should be done to reduce both the target volume extent and reduce the integral dose. In youngest patients, the possibility to use brachytherapy should be discussed to decrease integral dose in patient;

- in such a setting, proton therapy has been more and more proposed. The obvious balistic advantages of protons "may far outweigh the still hypothetical second cancer risk from particle radiotherapy in pediatric radiotherapy" (Trott, 2017).

\subsection{Adapting to specific organs}

All organs do not demonstrate an equal risk of a secondary radio-induced cancer. Some of them, such as the small intestine, are less sensitive to cancer radio-induction, while thyroid and breast are examples of organs highly sensitive to radiocarcinogenesis, a feature highly amplified by the age factor (with a high susceptibility in children) (Hall, 2006).

\subsection{Imaging dose management}

- IGRT (image guided radiotherapy) has become compulsory when using newly available highly precise treatment technologies. However, it brings an additional dose which should not be ignored;

- overdoses up to $8-10 \%$ have been reported accidentally with unadapted portal imaging systems and everyday controls (Peiffert et al., 2007). Radiation oncologists should be aware of the dose delivered by the IGRT they are using, and should adapt the number of controls to each patient's case.

\subsection{Other procedures to reduce the risk of radio-induced cancers}

They are based on procedures aiming at reducing as much as possible the "Integral dose", and have been presented in detail in the AAPM TG 158 document (Kry et al., 2017):

- avoidance of physical (or mechanical) wedges, responsible for an out-of-field dose higher by a factor of 2-4 relative to an open field;

- use of tertiary Multi leaf collimators (MLC);

- choice of the beam angles;

- jaws tracking;

- patient shielding;

- and accelerator shielding are other solutions to reduce the integral dose.

\section{Conclusions}

Even if radio-induced cancers are rare, they must be kept in mind each time a radiotherapy is proposed.

It has been pointed out that new technologies, such as IMRT, were responsible for an increase in the doses received out of the field. Fortunately, such a dose increase at distance is largely offset by the very significant reduction of the areas receiving high doses (areas where the risk of radiocarcinogenesis is higher).

Finally, even if new technologies were not considered to cause more second radio-induced cancers than conventional techniques, a continual effort should be made to reduce the outof-field doses delivered to patient(s) as a continued radiotherapy improvement strategy, thus following previous ICRP recommendations concerning optimization.

Age is one of the key parameters impacting on the risk of radio-induced secondary malignancies. Children could be nearly 3 to 6 times more sensitive to the carcinogenic effect of radiation than adults. Consequently, all efforts should be made to reduce the risk in children. In contrast, the second cancer risk is much lower, or even nil, in the elderly. In between, the secondary cancer risk, although most often low, should be kept in mind when designing therapeutic schemes and/or prescribing a specific irradiation.

\section{References}

Berrington de Gonzalez AB, Curtis RE, Kry SF, Gilbert E, Lamart S, Berg CD, Stovall M, Ron E. 2011. Proportion of second cancers attributable to radiotherapy treatment in adults: a cohort study in the US SEER cancer registries. Lancet Oncol. 12: 353-360.

Chargari C, Goodman KA, Diallo I, Guy JB, Rancoule C, Cosset JM, Deutsch E, Magne N. 2016. Risk of second cancers in the era of modern radiation therapy: does the risk/benefit analysis overcome theoretical models? Cancer Metastasis Rev. 35(2): 277-288.

Colin C, Foray N, Di Leo G, Sardanelli F. 2017. Radiation induced breast cancer risk in BRCA mutation carriers from low-dose radiological exposures: a systematic review. Radioprotection 52: 231-240.

Cosset JM, Henry-Amar M, Dietrich PY, Socié G, Girinsky T, Hayat M, Tubiana M. 1992. Tumeurs solides secondaires après radiothérapie de la maladie de Hodgkin: expérience de l'institut Gustave-Roussy. Bull. Cancer 79(4): 387-388. 
Cosset JM, Chargari C, Demoor C, Giraud P, Helfre S, Mornex F, Mazal A. 2016. Prévention des cancers radio-induits. Cancer Radiother. 20(Suppl.): S61-S68.

Foray N, Bourguignon M, Hamada N. 2016. Individual response to ionizing radiation. Mutat. Res. 770: 369-386.

Gray LH. 1957. Radiobiology and cancer. Nature 179(4568): 991994.

Hall E. 2006. Intensity-modulated radiation therapy, protons, and the risk of second cancers. Int. J. Radiat. Oncol. Biol. Phys. 65(1): $1-7$.

Koshy M et al. 2012. Declining use of radiotherapy in stages I and II Hodgkin's disease and its effect on survival and secondary malignancies. Int. J. Radiat. Oncol. Biol. Phys. 82: 619-620.

Kry SF, Bednarz B, Howell RM, Dauer L, Followill D, Klein E, Paganetti H, Wang B, Wuu CS, George Xu X. 2017. AAPM TG 158: measurement and calculation of doses outside the volume from external-beam radiation therapy. Med. Phys. 44(10): e391-e429.

Langballe R, Olsen JH, Andersson M, Mellemkjær L. 2011. Risk for second primary non-breast cancer in pre- and postmenopausal women with breast cancer not treated with chemotherapy, radiotherapy or endocrine therapy. Eur. J. Cancer 47: 946-952.

Little MP. 2001. Comparison of the risks of cancer incidence and mortality following radiation therapy for benign and malignant disease with the cancer risks observed in the Japanese A-bomb survivors. Int. J. Radiat. Biol. 77(4): 431-464.
Peiffert D, Simon JM, Eschwege F. 2007. Epinal radiotherapy accident: passed, present, future. Cancer Radiother. 11(6-7): 309-312.

Schneider U. 2011. Modeling the Risk of secondary malignancies after radiotherapy genes. Basel 2(4): 1033-1049.

Schneider U, Walsh L. 2008. Cancer risk estimates from the combined Japanese A-bomb and Hodgkin cohorts for doses relevant to radiotherapy. Radiat. Environ. Biophys. 47(2): 253-63.

Somers R, Henry-Amar M, Meerwaldt JH, Carde P. 1990. Treatment strategy in Hodgkin's disease. John Libbey Eurotext.

Suit $\mathrm{H}$, et al. 2007. Secondary carcinogenesis in patients treated with radiation: a review of data on radiation-induced cancers in human, non-human primate, canine and rodent subjects. Radiat. Res. 167: $12-42$.

Travis LB, et al. 2002. Lung cancer following chemotherapy and radiotherapy for Hodgkin's disease. J. Natl. Cancer Inst. 94(3): 182-192.

Trott KR. 2017. Special radiobiological features of second cancer risk after particle radiotherapy. Phys. Med. 42: 221-227.

Tubiana M. 2009. Can we reduce the incidence of second primary malignancies occurring after radiotherapy? A critical review. Radiother. Oncol. 91(1): 4-15.

Zelefsky MJ, et al. 2012. Incidence of secondary cancer development after high-dose intensity-modulated radiotherapy and imageguided brachytherapy for the treatment of localized prostate cancer. Int. J. Radiat. Oncol. Biol. Phys. 83(3): 953-959.

Cite this article as: Cosset J-M, Hetnal M, Chargari C. 2018. Second cancers after radiotherapy: update and recommandations. Radioprotection 53(2): 101-105 